\title{
Controlling a biological invasion: a non-classical dynamic economic model
}

\author{
Lars J. Olson · Santanu Roy
}

Received: 3 January 2007 / Accepted: 17 August 2007 / Published online: 25 September 2007

(C) Springer-Verlag 2007

\begin{abstract}
This paper analyzes the optimal intertemporal control of a biological invasion. The invasion growth function is non-convex and control costs depend on the invasion size, resulting in a non-classical dynamic optimization problem. We characterize the long run dynamic behavior of an optimally controlled invasion and the corresponding implications for public policy. Both control and the next-period invasion size may be non-monotone functions of the current invasion size; the related optimal time paths may not be monotone or convergent. We provide conditions under which eradication, maintenance control, and no control are optimal policies.
\end{abstract}

Keywords Intertemporal allocation · Nonconvexities · Biological invasion · Invasive species $\cdot$ Renewable resource economics

JEL Classification D9 - Q2

\section{Introduction}

Biological invasion - the invasion of ecological systems by non-indigenous species, is a very important component of global environmental change (Vitousek et al. 1996) and imposes significant economic and ecological damages. In the United States, the number of harmful invasive species is in the thousands and the total costs of

\footnotetext{
L. J. Olson $(\varangle)$

Department of Agricultural and Resource Economics, University of Maryland, College Park, MD 20742, USA

e-mail: lolson@arec.umd.edu

S. Roy

Department of Economics, Southern Methodist University,

Dallas, TX 75275, USA
} 
non-indigenous species have been estimated to be at least $\$ 137$ billion per year (Pimentel et al. 2000). Approximately one-fourth of the value of the country's agricultural output is lost to non-indigenous plant pests or the costs of controlling them (Simberloff 1996). Invasive species also cause significant ecological harm. They can alter ecosystem processes, act as vectors of disease, reduce biodiversity, and cause extinction of other organisms (Vitousek et al. 1996).

An invasive species is a natural resource whose biological capital stock causes economic damage. The purpose of this paper is to examine the economics of controlling a biological invasion where the objective is to minimize the discounted sum of social costs associated with invasion damages and control costs. We confine attention to invasive pest species that provide no net consumptive benefits. ${ }^{1}$ The aim is to understand the economic and biological conditions that determine when an invasion should be controlled, when it should be eradicated, and when it is optimal to do nothing.

This paper contributes to the literature on optimal capital accumulation in a onesector dynamic economic model that includes models of optimal economic growth as well as optimal management of renewable resources. The classical model in this literature involves maximizing the discounted sum of social welfare generated through consumption, with productive possibilities determined by a physical or biological production function. A "non-classical" strand in this literature characterizes the optimal dynamic policy in models where the production function is non-concave so that the feasible set is non-convex [see, among others, Clark (1990), Majumdar and Mitra (1982), Dechert and Nishimura (1983) and Mitra and Roy (2006)] as well as models where the utility function depends on both capital stock and consumption [see, for example, Majumdar and Mitra (1994), Nyarko and Olson (1994), Olson and Roy (1996) and the collection of papers in Majumdar et al. (2000)]. Dawid and Kopel (1999) consider the case where the feasible set is convex but the objective function is non-concave.

At first glance, it might seem that the problem of controlling a biological invasion is isomorphic to one of these standard intertemporal capital accumulation problems. This is not the case, however, and maximizing the negative of the discounted stream of costs does not suffice to transform our problem into a standard optimal growth or resource management problem. There are three reasons for this. First, an increase in the stock of an invasive species reduces intertemporal welfare. Hence, the shadow price of the biological capital stock is negative whereas in a standard model of capital accumulation it is positive. Second, the set of attainable welfare levels becomes smaller as the size of the capital stock increases. This is in contrast to a typical model of optimal growth or resource management where a larger capital stock is associated with a larger set of attainable welfare levels. Third, unlike the problem of optimal growth or resource management, the dynamic optimization problem for controlling an invasive species is convex when the biological growth (or production) function is convex. As no biological invasion can expand indefinitely, the biological growth function must be bounded and

\footnotetext{
1 Unlike biological species with positive consumption value, the harvest or control of harmful species frequently occurs through direct actions of central or local public authorities. Our analysis offers insight into dynamically efficient public programs for controlling such species, whether implemented through public management or through incentive based decentralized instruments.
} 
therefore, non-convex. This implies that the transition equation for the invasive species problem is necessarily non-convex.

The cost of control in the invasive species problem is the minimum cost of achieving a given reduction in the invasion size through efficient use of chemical, biological, mechanical, manual and other control inputs. It typically costs less to achieve a given amount of control from a large invasion than it does from a small invasion so that the cost of control depends on both the amount controlled (the reduction in the size of the invasion) and on the size of the invasion being controlled (the capital stock). This means that the immediate return function depends on both the state and control. As there seems to be scant justification for assuming that the control cost is jointly convex in both the amount controlled and the invasion size, we make no such assumption. Thus, our paper can be seen as a model of one-sector optimal capital accumulation that encompasses non-convexities and state-dependence in the objective function, a non-convex production technology, and a negative shadow price of capital. To our knowledge there is no existing paper that simultaneously considers these non-classical features.

The extant literature on the dynamic economics of invasive species control is small. An early precursor and the most closely related paper is Jaquette's (1972) analysis of a discrete time biological population control model. Jaquette's analysis only considers the existence of an optimal policy and the monotonicity of the optimal state transition in a finite horizon problem. We examine the infinite horizon case in a model that admits both monotone and non-monotone policies, and we provide a more complete characterization of the different outcomes in terms of the economic and ecological fundamentals of the model.

In an earlier paper (Olson and Roy 2002) we examined a stochastic model where control costs are independent of the invasion size. The latter assumption is too restrictive to be useful as a positive theory in many applications. In addition, it has the normative implication that the optimal state transition function is always monotonic. This rules out an interesting class of situations where, as an invasion grows larger, the amount controlled increases at a faster rate than the increase in invasion size. The current paper characterizes the dynamics of an optimally controlled invasion for the more realistic and richer class of intertemporal allocation problems where control costs depend on the state and where the optimal path of invasion size need not be monotonic or convergent.

The main contribution of this paper is twofold. First, it brings out precisely the joint implications of non-convex biological growth and state dependent control costs for the dynamics of an optimally controlled biological invasion and its long run behavior. Second, it characterizes the limiting outcomes under an optimal policy by examining conditions under which the optimal policy leads to: (i) eradication of an invasion from any size, (ii) eradication of small invasions but not large invasions, and (iii) non-eradication. These conditions are given in terms of the ecological and economic fundamentals of the model: the biological growth of the invasion, control costs, damages and the social discount rate.

The analysis of dynamic optimality of (immediate or eventual) eradication and non-eradication of an invasive species is closely related to the analysis of optimality of extinction of renewable resources and physical capital stocks in the existing literature. 
However, for a harmful invasive species, the qualitative factors that make it optimal to eradicate the species (such as a high natural growth rate or productivity and mild discounting of future welfare) are precisely the ones that make non-extinction (or conservation) optimal for beneficial renewable resources or physical capital. Further, the precise conditions under which eradication of an invasive species is optimal or not optimal cannot be derived from existing conditions for conservation and extinction in comparable non-classical models of renewable resource management with stockdependent reward function and non-concave biological growth function [such as Olson and Roy (1996)]. Indeed (as we explain at the end of Sect. 6), there are inherent, qualitative differences between the kind of conditions that make conservation optimal in a renewable resource model and the conditions that make eradication optimal in our framework.

The paper is organized as follows. Section 2 develops the model. The basic optimality conditions that govern a controlled invasion are derived in Sect. 2. We use Dini derivatives to extend the envelope theorem of Mirman and Zilcha (1975) and Benveniste and Scheinkman (1979) to the case where the objective function and feasible set of time paths are non-convex. Sections 3 and 4 examine conditions under which it is optimal to control a biological invasion and conditions under which no control is optimal. The main results on the economics of eradication are presented in Sects. 5 and 6. Concluding remarks follow. All proofs are in the appendix.

\section{The model and its basic properties}

Let $y_{t}$ represent the invasion size at time $t$ and let $a_{t}$ represent the control, or reduction in the invasion size undertaken at time $t$, where $0 \leq a_{t} \leq y_{t}$. The units of measurement for the invasion size may vary depending on the context. For example, the invasion size may be measured by population, biomass, or the area under invasion. The invasion size that remains following control is $x_{t}=y_{t}-a_{t}$. The invasion grows and spreads over time according to a natural, ecological production function $y_{t+1}=f\left(x_{t}\right)$. The $t$ th iterate of $f(\cdot)$ and its derivative are defined by $f^{0}(x)=x, f^{t}(x)=f^{t-1}(f(x)), t=1, \ldots$ and $f_{x}^{t}(x)=\frac{\mathrm{d} f^{t}(x)}{\mathrm{d} x}$. The production function is assumed to satisfy the following properties:

A1. $f: \Re_{+} \rightarrow \Re_{+}$is continuously differentiable on $\Re_{++}$, with the derivative denoted by $f_{x}(x)$.

A2. $f(0)=0$.

A3. $f_{x}(x) \geq 0$.

A4. $f_{x}(0)=\liminf _{x \rightarrow 0} f_{x}(x)>1$.

A5. There exists $K \in(0, \infty)$ such that $f(x)<x$ for all $x>K, f(K)=K$ and $f_{x}(x)>0$, for all $x \in[0, K]$.

In this paper we do not consider new introductions of an invasive species. Under assumption A2 an invasion cannot regenerate once it has been eradicated. Assumption A3 says that the invasion production function is weakly increasing. Assumption A4 insures that there exists a feasible steady state and that, in the absence of control, the invasion is sustainable in the long run. Assumption A5 implies that the invasion size is bounded. 
We do not assume convexity of $f$. Indeed, every bounded invasion necessarily has a non-convex production function and non-convexities are a ubiquitous feature of invasive species management problems. This has both positive and normative implications. The marginal benefit from a reduction in the invasion size may decrease as the invasion becomes larger, contrary to what might be expected. As a consequence, the optimal control policy may be a non-monotone function of the invasion size and it may be optimal to reduce control as the invasion size increases. Observing a negative relation between control and the invasion size is not necessarily indicative of sub-optimal policy.

The immediate costs of control are denoted by $C(a, y) . C(a, y)$ represents the minimum cost over the set of feasible inputs that can reduce the invasion size by an amount equal to $a$, when the current invasion size is $y$. When the amount controlled, $a$, is viewed as the output of control activities and $y$ is viewed as a fixed input, then $C(a, y)$ is a standard cost function for the production of $a$, where the prices of control inputs have been supressed in the cost function. The invasion size that remains after control, $x=y-a$, causes social damages $D(x)$. Typically these damages arise because the invasive species adversely impacts the production of other goods, as in the case of agricultural pests, or because the invasive species has negative effects on amenities that yield social welfare.

The following notation is used throughout the paper. Derivatives are indicated by relevant subscripts, e.g., $C_{a}$ represents the partial derivative of $C$ with respect to $a$. Let $\Omega \subset \Re_{+}^{2}$ be defined by: $\Omega=\{(a, y): 0 \leq a \leq y \leq K\}$. Costs and damages are assumed to satisfy the following:

B1. C: $\Omega \rightarrow \Re_{+}$and $D: \Re_{+} \rightarrow \Re_{+}$are twice continuously differentiable.

B2. $C(0, y)=0$ for all $y \geq 0$. $D(0)=0$.

B3. $C_{a}(a, y) \geq 0, C_{y}(a, y) \leq 0$, and $C_{a}(a, y)+C_{y}(a, y) \geq 0$ on $\Omega$. $D_{x}(x) \geq 0$.

B4. For any $y \geq 0, C(a, y)$ is convex in $a$ on $[0, y]$. $D$ is convex on $\Re_{+}$.

Assumption B2 rules out fixed costs and it implies that $C_{y}(0, y)=0$ for all $y$. Assumption B3 implies that damages are increasing, that the costs of control increase as control increases, and that a given reduction in the invasion size is cheaper to achieve from a larger invasion than a smaller one. The assumption that $C_{a}(a, y)+$ $C_{y}(a, y) \geq 0$ means that if $y \leq y^{\prime}$ then for any $x \leq y$ it is less costly to reduce the invasion size from $y$ to $x$ than it is to reduce it from $y^{\prime}$ to $x$. Assumption B4 requires that for any invasion size, marginal control cost is non-decreasing in the amount of control and marginal damage is non-decreasing in the size of invasion that is left uncontrolled. Assumptions A1-A5 and B1-B4 are assumed to hold throughout the paper.

Note that we do not assume that $C$ is jointly convex in $a$ and $y$. There is little evidence to support the hypothesis that joint convexity $C(a, y)$ is a universal property of invasive species control problems. For example, if the marginal cost of control is more sensitive to changes in the invasion size than it is to changes in control then the control cost function must be nonconvex. This type of nonconvexity is present in the model of Rondeau and Conrad (2003), for example.

The objective of the dynamic optimization problem is to minimize the discounted sum of control costs and social damages over time subject to the production function 
that governs the growth and spread of the invasion. The optimal value function or social cost function is:

$$
\begin{aligned}
V\left(y_{0}\right)= & \min \sum_{t=0}^{\infty} \delta^{t}\left[C\left(a_{t}, y_{t}\right)+D\left(x_{t}\right)\right] \\
& \text { subject to : } y_{t}=a_{t}+x_{t}, y_{t+1}=f\left(x_{t}\right)
\end{aligned}
$$

where $y_{0}$ represents the initial stock. Under A1-A5 and B1-B4, standard dynamic programming arguments imply that there exists a stationary optimal value function that satisfies the recursion

$$
V\left(y_{t}\right)=\min _{0 \leq x_{t} \leq y_{t}, a_{t}=y_{t}-x_{t} .}\left[C\left(a_{t}, y_{t}\right)+D\left(x_{t}\right)+\delta V\left(f\left(x_{t}\right)\right)\right]
$$

and that there exists a stationary Markov optimal policy whose decision rules are $X(y)=\arg \min \{C(y-x, y)+D(x)+\delta V(f(x)): 0 \leq x \leq y\}$ and $A(y)=$ $y-X(y)$ [see Strauch (1966)]. A sequence $\left\{y_{t}, x_{t}, a_{t}\right\}_{t=0}^{\infty}$ that solves (1) is an optimal program from $y_{0}$. Given an initial invasion size $y_{0}=y$ and a selection $x(y)$ from the stationary optimal policy $X(y)$, an optimal program is defined recursively by $y_{t+1}=f\left(x\left(y_{t}\right)\right), x_{t}=x\left(y_{t}\right), a_{t}=y_{t}-x\left(y_{t}\right), t=0,1,2, \ldots$

Assumption B3 implies that a larger invasion imposes larger social costs.

\section{Lemma 2.1 $V(y)$ is continuous and non-decreasing.}

When control costs depend on the invasion size an increase in control has two opposing effects on future net benefits. First, it lowers future damages. Second, by reducing the invasion size, it increases future control costs. If marginal costs of control are very sensitive to changes in the invasion size then the qualitative dynamics of optimal paths can be affected. Let $x \in X(y)$ and $x^{\prime} \in X\left(y^{\prime}\right)$ where $y \leq y^{\prime}$. The correspondence $X(y)$ is an ascending correspondence if $\min \left\{x, x^{\prime}\right\} \in X(y)$ and $\max \left\{x, x^{\prime}\right\} \in X\left(y^{\prime}\right)$. Similarly, $X(y)$ is descending if $\max \left\{x, x^{\prime}\right\} \in X(y)$ and $\min \left\{x, x^{\prime}\right\} \in X\left(y^{\prime}\right)$.

Lemma 2.2 If $C_{a a}(a, y)+C_{a y}(a, y) \geq 0$ on $\Omega$, then $X(y)$ is an ascending correspondence and the maximal and minimal selections from $X$ are non-decreasing functions. If the inequality is strict then every selection from $X$ is non-decreasing.

Note that if we write the control cost as a function of initial invasion size and the size of the invasion left after control, $\phi(x, y)=C(y-x, y)$, then the requirement that $C_{a a}(a, y)+C_{a y}(a, y) \geq 0$ is equivalent to the requirement that the second order cross partial derivative of $\phi$ is non-positive; that is, the control cost function is submodular in $(x, y)$. We shall therefore refer to the case where $C_{a a}(a, y)+C_{a y}(a, y) \geq 0$ as the "submodular case".

If $x(y)$ is non-decreasing then the optimal transition function, $f(x(y))$, is also nondecreasing. Hence, the optimal path $\left\{y_{t}\right\}$ is monotonic over time and $y_{t}$ converges to an optimal steady state as $t \rightarrow \infty$, that may not be unique. When there are multiple optimal steady states, the limiting outcome depends on the initial invasion size. 
If the marginal cost of control is more sensitive to changes in the invasion size than changes in control so that $C_{a a}+C_{a y}<0$ (i.e., control cost is supermodular in $(x, y)$ ) over some part of the domain, then $X(y)$ may be non-monotonic in $y$. The optimal path may exhibit cyclic or chaotic dynamics and the optimal policy may involve periodic control where the invasion size is allowed to grow unchecked for a number of periods until it becomes large enough that control is efficient. Once the state is reduced, it is allowed to grow again and the process repeats itself.

The final result of this section characterizes the intertemporal trade-offs between marginal costs and damages along an optimal program.

Proposition 2.3 Let $\left\{y_{t}, x_{t}, a_{t}\right\}_{t=0}^{\infty}$ be an optimal program. (a) If $a_{t}>0$ then $C_{a}\left(a_{t}, y_{t}\right)$ $\leq D_{x}\left(x_{t}\right)+\delta\left[C_{a}\left(a_{t+1}, y_{t+1}\right)+C_{y}\left(a_{t+1}, y_{t+1}\right)\right] f_{x}\left(x_{t}\right)$. (b) If $x_{t}>0$ and $a_{t+1}>0$ then $C_{a}\left(a_{t}, y_{t}\right) \geq D_{x}\left(x_{t}\right)+\delta\left[C_{a}\left(a_{t+1}, y_{t+1}\right)+C_{y}\left(a_{t+1}, y_{t+1}\right)\right] f_{x}\left(x_{t}\right)$. (c) If $0<x_{t}<y_{t}$ and $a_{t+1}>0$ then $C_{a}\left(a_{t}, y_{t}\right)=D_{x}\left(x_{t}\right)+\delta\left[C_{a}\left(a_{t+1}, y_{t+1}\right)+C_{y}\left(a_{t+1}, y_{t+1}\right)\right] f_{x}\left(x_{t}\right)$ (d) If $0<x_{t}<y_{t}$ for all $t$ then $C_{a}\left(a_{t}, y_{t}\right)=D_{x}\left(x_{t}\right)+\sum_{i=1}^{\infty} \delta^{i}\left[D_{x}\left(x_{t+i}\right)+\right.$ $\left.C_{y}\left(a_{t+i}, y_{t+i}\right)\right] \prod_{j=0}^{i-1} f_{x}\left(x_{t+j}\right)$.

The last condition has a simple interpretation when the costs of control are independent of the invasion size. For an interior policy the optimal control must satisfy an intertemporal cost-benefit criterion that balances the marginal cost of control against with the discounted sum of marginal damages times the compounded marginal productivity of the invasion associated with the last unit that remains uncontrolled.

Throughout the paper the following example will be used to illustrate our results.

Example 2.1 Costs and damages are exponential and given by $C(a, y)=(\exp (\alpha a)-$ 1) $\exp (-\beta y)$ and $D(x)=\exp (\gamma x)$.

In this example, the parameter $\alpha$ represents the intrinsic marginal cost of control, or the marginal cost of control evaluated at the limiting values of $a=0$ and $y=$ 0 . Similarly, $\gamma$ represents intrinsic marginal damage, or marginal damage from an incrementally small invasion. Assumption B.3 requires $\frac{\alpha}{\beta}>1-\exp (-\alpha K)$. Control cost is submodular (supermodular) as $\alpha>(<) \beta$. Hence, the optimal policy governing the invasion size is monotone if $\alpha>\beta$, while if $\beta>\alpha$ the optimal invasion size is governed by a decreasing policy on the interior of $\Omega$.

\section{When is it optimal to control?}

The question of when to control is one of the fundamental questions in a dynamic control problem. This is true even in a convex model (Inada 1963), but it is especially relevant in nonconvex models where corner solutions are more likely to be optimal. In our context there may be some problems where control is not cost effective because damages are small relative to control costs. In other cases control may only be worthwhile if the invasion size is large enough to cause significant damages. Finally, there will exist problems where it is always optimal to control. We differentiate between three cases. 
Definition (a) The system is eventually controlled from $y$ if there exists some $T$ such that $A\left(y_{T}\right)>0$, where $\left\{y_{t}\right\}$ is an optimal program from $y_{0}=y$. (b) The system is controlled from $y$ if $A(y)>0$. (c) The system is globally controlled if $A(y)>0$ for all $y>0$.

Each successive definition of control is more restrictive in the sense that (c) $\Longrightarrow$ (b) $\Longrightarrow$ (a). We now characterize the economic conditions that are sufficient for different types of control. Proposition 3.1 involves comparisons of the marginal cost of control with the discounted marginal social costs associated with a policy that delays control for a given number of periods.

Proposition 3.1 (a) If there is $n \geq 0$ such that $C_{a}\left(0, f^{n}(y)\right)<\sum_{i=0}^{\infty} \delta^{i}\left[D_{x}\left(f^{i}(y)\right)\right.$ $\left.f_{x}^{i}(y)\right]$ then the system is eventually controlled from $y>0$. (b) If the system is eventually controlled from $y>0$ and if $C_{a}(0, y)<\inf _{t \geq 1}\left\{\sum_{k=0}^{t-1} \delta^{k} D_{x}\left(f^{k}(y)\right) f_{x}^{k}(y)+\right.$ $\left.\delta^{t} \inf _{a}\left[C_{a}\left(a, f^{t}(y)\right)+C_{y}\left(a, f^{t}(y)\right)\right] f_{x}^{t}(y)\right\}$ then the system is controlled from $y$. (c) If $C_{a}(0, y)<D_{x}(y)+\delta\left[\inf _{a}\left\{C_{a}(a, f(y))+C_{y}(a, f(y))\right\}\right] f_{x}(y)$ for all $y>0$ then the system is globally controlled.

In the case where $C_{a a}+C_{a y} \geq 0$ on $\Omega$, the condition in Proposition 3.1(b) simplifies to $C_{a}(0, y)<\inf _{t \geq 1}\left\{\sum_{k=0}^{t-1} \delta^{k} D_{x}\left(f^{k}(y)\right) f_{x}^{k}(y)+\delta^{t} C_{a}\left(0, f^{t}(y)\right) f_{x}^{t}(y)\right\}$.

From Proposition 3.1(b) it is easy to see that if control is cost-effective for a myopic planner, or $C_{a}(0, y)<D_{x}(y)$, then control is also cost-effective for a planner who optimizes over time. If the inequality in part (b) is true for all $y>0$, then it is always optimal to control the system, no matter what the invasion size is.

With exponential costs and damages as outlined in Example 2.1, eventual control is optimal if $\alpha \exp \left(-\beta f^{n}(y)\right)<\gamma \sum_{t=0}^{\infty} \delta^{t} \exp \left(\gamma f^{t}(y) f_{x}^{t}(y)\right.$ for some $n$. If $\alpha>\beta$, control from $y$ is optimal whenever $\alpha \exp (-\beta y)<\inf _{t \geq 1} \gamma \sum_{i=0}^{t-1} \delta^{i} \exp \left(\gamma f^{i}(y) f_{x}^{i}(y)\right.$ $+\alpha \delta^{t} \exp \left(-\beta f^{t}(y)\right) f_{x}^{t}(y)$ and global control is optimal if $\alpha \exp (-\beta y)<\gamma \exp (\gamma y)+$ $\delta \alpha \exp (-\beta f(y)) f_{x}(y)$ for all $y$. When $\beta>\alpha$, control from $y$ is optimal if $\alpha \exp (-\beta y)$ $<\inf _{t \geq 1} \gamma \sum_{i=0}^{t-1} \delta^{i} \exp \left(\gamma f^{i}(y) f_{x}^{i}(y)+\delta^{t} \exp \left(-\beta f^{t}(y)\right)\left[\beta+(\alpha-\beta) \exp \left(\alpha f^{t}(y)\right]\right.\right.$ $f_{x}^{t}(y)$, while global control is optimal if $\alpha \exp (-\beta y)<\gamma \exp (\gamma y)+\delta \exp (-\beta f(y))$ $\left[\beta+(\alpha-\beta) \exp (\alpha f(y)] f_{x}(y)\right.$ for all $y$. The difference between the two cases $\alpha>\beta$ and $\beta>\alpha$ is the last term on the right side of the inequality. This term represents the marginal cost of control in the future period when control is resumed. It is evaluated at different levels of control, depending on whether cost is submodular or supermodular.

\section{When is no control optimal?}

In a nonconvex model it is equally important to understand the conditions under which current control is not cost-effective, either because control is too costly relative to damages or because it is better to wait and control at a later date. Proposition 4.1 implies that there should be no control in the current period if the marginal costs of initiating control exceed the maximum current and future marginal damages that can occur, given the invasion size. Since marginal damages increase with the invasion size, the proposition is less likely to hold for larger invasions. 
Proposition 4.1 If $C_{a}(0, y)>D_{x}(y)+\delta\left[\sup _{\substack{0 \leq a \leq f(x) \\ 0 \leq x \leq y}}\left(C_{a}(a, f(x))+C_{y}(a, f(x))\right]\right.$ $f_{x}(x)$, then $A(y)=0$ and $X(y)=y$.

In Example 2.1, it is optimal not to control from current invasion size $y>0$, when $\alpha>$ $\beta$ and $\alpha \exp (-\beta y)>\gamma \exp (\gamma y)+\delta \sup _{0 \leq x \leq y} \exp (-\beta f(x))[\beta+(\alpha-\beta) \exp (\alpha f(x)]$ $f_{x}(x)$, or when $\beta>\alpha$ and $\alpha \exp (-\beta y)>\gamma \exp (\gamma y)+\delta \sup _{0 \leq x \leq y} \exp (-\beta f(x)) f_{x}(x)$.

The next proposition is a stronger result that examines when it is optimal to refrain from control if the invasion size is small. However, it only applies when control cost is submodular. The discounted intrinsic growth rate must be less than one so the invasion must exhibit low biological productivity from small invasion sizes. In addition, marginal damages compounded indefinitely at the discounted intrinsic growth rate must be less than the marginal costs of control. When these two conditions are satisfied then the optimal policy is not to control when the invasion size is sufficiently small.

Proposition 4.2 If $C_{a a}(a, y)+C_{a y}(a, y) \geq 0$ on $\Omega$ and $D_{x}(0)+\delta C_{a}(0,0) f_{x}(0)<$ $C_{a}(0,0)$ then for all $y$ sufficiently close to zero, $A(y)=0$ and $X(y)=y$.

In practice, this only requires an evaluation of marginal costs and damages at the origin, or intrinsic marginal costs and damages, along with the intrinsic growth rate of the invasion, $f_{x}(0)$. Rearranging terms, it can be seen that the proposition holds if $\delta f_{x}(0)<1-\frac{D_{x}(0)}{C_{a}(0,0)}$, that is, when the discounted intrinsic growth of the invasion is less than one minus the ratio of intrinsic marginal damages to intrinsic marginal costs. In this case the invasion is growing too slowly to warrant control from small invasion sizes. With exponential cost and damage as outlined in Example 2.1, this condition simply requires that $\alpha>\gamma+\delta \alpha f_{x}(0)$.

Proposition 4.2 comes close to providing a necessary and sufficient condition for no control to be optimal from small invasion sizes. This can be seen by a comparison of Proposition 4.2 with Proposition 3.1(c) evaluated as the invasion size approaches zero.

\section{The economics of eradication}

In this section, we consider the conditions under which it makes sense to eradicate an invasive species, where eradication is defined as $\limsup _{t \rightarrow \infty}\left\{y_{t}\right\}=0$. Eradication requires that that the invasion be controlled and therefore, all propositions in this section require that the invasion is controlled under an optimal policy over relevant ranges of the state variable. A sufficient condition for this is given in Proposition 3.1(b).

It is intuitively clear that a necessary step toward eradication is a reduction in the size of invasion.

Proposition 5.1 Suppose the invasion is controlled from all $y^{\prime} \in[y, f(y)]$ and, that for all $x \in\left(f^{-1}(y), y\right)$,

$$
C_{a}(y-x, y)<D_{x}(x)+\delta\left[\inf _{0 \leq a \leq f(x)-x} C_{a}(a, f(x))+C_{y}(a, f(x))\right] f_{x}(x) .
$$

Then every optimal program from $y_{0}=y$ satisfies $y_{1}=f\left(x_{0}\right)<y_{0}$. 
To interpret this result, first examine the static case where $\delta=0$. Consider the set of controls that produce an increase in the size of the invasion. If marginal damages exceed marginal costs for every control in this set, then it can never be optimal to allow the invasion to increase in size. In the dynamic case, marginal control costs are compared to the current and future marginal damages, adjusted for the effect of the invasion size on future control costs. The second term on the right hand side of the inequality in Proposition 5.1 is a lower bound on the effect that a reduction in the invasion today has on future damages and control costs.

We now characterize the economic and biological conditions under which eradication is optimal. In general, this depends on the initial size of the invasion and there may exist a threshold, $\zeta$, such that eradication is optimal when the invasion size is less that $\zeta$, but if the invasion size is larger than $\zeta$ then eradication is no longer optimal. When discounted marginal productivity exceeds one from every invasion size below $\zeta$, then eradication is optimal even if current marginal damages are very low. The rationale is that a fast growing invasion increases future damages and control costs, and if these costs increase more rapidly that the rate of discount then it makes sense not to allow the invasion to grow and the invasion should be eradicated from its current level. The condition for eradication becomes stronger with higher values of $\zeta$. Thus, eradication is more likely to be optimal from small invasions than from large invasions.

If control cost is submodular, the efficiency of eradication depends on the economic and biological conditions at potential steady states and a tighter condition for eradication is possible. The condition in Proposition 5.2(b) balances the marginal costs of steady state control against the current marginal damages plus the lower bound on future marginal damages and control costs. If the current and future marginal damages from steady state control are higher for all invasions smaller than $\zeta$ then eradication is optimal from all such invasions.

If $\zeta=K$ in part (a) or (b) of Proposition 5.2, then global eradication is optimal. However, Proposition 5.2(c) gives a more direct condition for global eradication by ensuring that there is no positive steady state.

Proposition 5.2 (a) If there exists $a \zeta>0$ such that the invasion is controlled from every $y \in(0, f(\zeta))$, and if (2) holds for every $y \in(0, \zeta]$ and for all $x \in$ $\left(f^{-1}(y), y\right)$, then eradication is optimal from every invasion of size $y \in(0, \zeta]$. (b) Assume $C_{a a}(a, y)+C_{a y}(a, y) \geq 0$ on $\Omega$. If there exists $a \zeta>0$ such that the invasion is controlled from every $y \in(0, f(\zeta)]$, and if $C_{a}(f(x)-x, f(x))<$ $D_{x}(x)+\delta C_{a}(0, f(x)) f_{x}(x)$ for all $x \in(0, \zeta]$, then eradication is optimal from every invasion of size $y_{0} \in(0, \zeta]$. (c) If $C_{a a}(a, y)+C_{a y}(a, y) \geq 0$ on $\Omega$ and if $C_{a}(f(x)-x, f(x))<D_{x}(x)+\delta\left[C_{a}(f(x)-x, f(x))+C_{y}(f(x)-x, f(x))\right] f_{x}(x)$ for all $x \in(0, K]$, then every optimal program converges to zero and eradication is globally optimal.

There are three differences between part (b) and (c) of the proposition. First, part (b) relies on a lower bound on future marginal damages while this is not necessary for (c). Second, part (b) can be used to evaluate the efficiency of eradication from small invasions; the conditions need not apply when the invasion is large. On the other hand, part (c) is a result about global eradication so the restrictions it imposes are required to hold for every possible invasion size. For the case of exponential cost and damage 
functions in Example 2.1, eradication is optimal from small invasions if $\alpha<\gamma+$ $\delta \alpha f_{x}(0)$. Eradication of large invasions requires $\alpha \exp (\alpha(f(x)-x)) \exp (-\beta f(x))<$ $\gamma \exp (\gamma x)+\delta \exp (-\beta f(x))[\beta+(\alpha-\beta) \exp (\alpha(f(x)-x))] f_{x}(x)$ for all $x$.

Next, we characterize the circumstances under which immediate eradication is optimal, i.e., where the invasion is fully eradicated in the current period.

Proposition 5.3 Suppose that for some $y \in(0, K]$, the invasion is controlled from every $y_{0} \in(0, f(y))$ and that $C_{a}(y, y)<D_{x}(0)+\delta \inf _{0 \leq a \leq f(x)-x}\left[\left(C_{a}(a, f(x))+\right.\right.$ $\left.\left.C_{y}(a, f(x))\right) f_{x}(x)\right]$. Then, immediate eradication is optimal from $y$.

The criterion for immediate eradication balances the costs of removing the last unit of the invasion against the current and future damages that would be caused should the invasion be allowed to remain. It is possible that the condition for immediate eradication may be satisfied for large $y$ and not for small $y$. That is, immediate eradication may be optimal for a large invasion even if it is not optimal for a smaller invasion. Since Propositions 5.1 and 5.3 are not mutually exclusive there can exist circumstances where: eradication of small invasions is optimal, eradication is not optimal for medium size invasions, and eradication is optimal for large invasions.

These results on the economics of eradication have the following implications. First, eradication is more likely to be an optimal policy for invasions that have higher discounted marginal productivity than it is for invasions that grow slowly. This might seem counter-intuitive, but it is because the benefits from control or future damages prevented are higher when an invasion expands rapidly. In addition, the benefits from current control are given greater weight in the future when the discount rate is low. Second, for some invasions economic considerations may favor eradication when the invasion is small, but not when the invasion is large. In such cases a rapid response may be necessary for eradication to be justified. Finally, in the special case where the marginal costs of control at $a=0$ are insignificant, the criteria for eradication in Propositions 5.1, 5.2(b) and 5.3 essentially involve static benefit cost considerations that balance current marginal costs and damages. This is a consequence of the fact

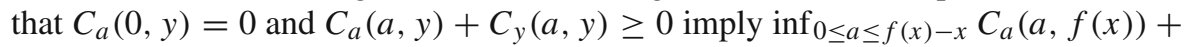
$C_{y}(a, f(x))=0$. Hence, the lower bound on future marginal social costs is relatively weak when marginal control cost is insignificant.

\section{The economics of noneradication}

In this section we characterize the economic and biological environments under which eradication is not optimal. The optimal policy either involves no intervention, or suppression in order to reduce damages. Our first result rules out immediate eradication as an optimal strategy. If the damages from an arbitrarily small invasion are less than the marginal costs of removing the entire invasion, then it is always optimal to allow some of the invasion to remain. Further, in the submodular case, eradication of a small invasion is not optimal if the damages compounded indefinitely at the discounted intrinsic growth rate are less than the marginal cost of eradicating the invasion. 
Proposition 6.1 (a) If $D_{x}(0)+\delta C_{a}(0,0) f_{x}(0)<C_{a}(y, y)$ then $X(y)>0$. (b) If $C_{a a}(a, y)+C_{a y}(a, y) \geq 0$ on $\Omega$ and $D_{x}(0)+\delta C_{a}(0,0) f_{x}(0)<C_{a}(0,0)$ then $X(y)>0$ for all $y$ and, in addition, for all $y$ sufficiently close to zero $A(y)=0$ and $X(y)=y$.

For the exponential cost and damage functions in Example 2.1, immediate eradication is not optimal if $\alpha \exp ((\alpha-\beta) y)>\gamma+\delta \alpha f_{x}(0)$. This does not rule out eradication as a future possibility. Eventual eradication is not optimal if $\alpha>\beta$ and $\alpha>\gamma+\delta \alpha f_{x}(0)$.

A final possibility is that eradication is optimal for a small invasion but not a large invasion. The last result can be used to help identify such outcomes.

Proposition 6.2 (a) Suppose there exists an $\widehat{x}$ such that every invasion of size $y \geq \widehat{x}$ is controlled and that $C_{a}(y-\widehat{x}, y)>D_{x}(\widehat{x})+\delta \sup _{0 \leq x<x<, 0 \leq a \leq f(x)}\left[\left(C_{a}(a, f(x))+C_{y}\right.\right.$ $\left.(a, f(x))) f_{x}(x)\right]$. Then from every initial invasion size $y \geq f(\widehat{x})$, the invasion size in every period along any optimal path is bounded below by $f(\widehat{x})$. (b) Assume $C_{a a}(a, y)+C_{a y}(a, y) \geq 0$ on $\Omega$. Suppose there exists an $\hat{x}$ such that for every $x \leq \widehat{x}, C_{a}(f(x)-x, f(x))>D_{x}(x)+\delta\left[C_{a}(f(x), f(x))+C_{y}(f(x), f(x))\right] f_{x}(x)$. Then from every initial invasion size $y \geq f(\widehat{x})$, the invasion size in every period along any optimal path is bounded below by $f(\widehat{x})$.

If the marginal costs of reducing the size of the invasion over time exceed the current and future marginal damages for every invasion larger than $f(\widehat{x})$, then it can never be efficient to reduce the invasion size below $f(\widehat{x})$. Note that as $C_{y}<0$, even if $\delta f_{x}(0)>1$ it is possible that non-eradication may be globally optimal. For a beneficial renewable resource with similar biological growth properties (with or without stockdependent harvesting cost and benefit), conservation is always optimal. This shows that even though one can think of eradication as the inverse of conservation, the conditions for eradication and non-eradication of harmful invasive species are inherently different from those associated with conservation of beneficial renewable resources.

\section{Conclusion}

The results of this paper can provide the foundation for both a normative and a positive analysis of invasive species control. As such, the results show the importance of the initial invasion size in determining the optimal policy. The paper also illustrates the information that is needed to evaluate the economic efficiency of invasive species control. Estimates of the costs of control, damages from an invasion, and the invasion production function or invasion growth rate are required. These may be difficult to assess, particularly in the early stages of an invasion. Yet this is precisely the time when prompt action can reduce future consequences.

There are a number of important issues related to invasive species problems that are not addressed in this paper. There are many circumstances where prevention may be the best control and our model does not consider the possibility of re-invasion. Clearly, the value of eradicating an invasive species will depend on the likelihood that a new invasion might occur. Finally, many invasive species problems involve private actions 
where individuals do not consider the consequences for social welfare. The design of policies that mitigate the conflicts between private incentives and social welfare is another interesting aspect of invasive species problems.

Acknowledgements We thank a referee for useful comments. This research was supported by USDAPREISM grant No. 433AEM380082.

\section{Appendix}

Proof of Lemma 2.1 The proof that $V$ is continuous is standard. The proof that $V$ is non-decreasing uses $A 3$ and $B 3$.

Proof of Lemma $2.2 C(y-x, y)+D(x)+\delta V(f(x))$ is submodular in $(y, x)$ so that arguments from Topkis (1978) apply. For details, see the working paper version (Olson and Roy 2006).

Proof of Proposition 2.3 To accomodate non-convexity and the possibility of corner solutions we use the Dini derivatives of $V$ to characterize the marginal optimality conditions. Since we are dealing with a one state variable problem, these exist everywhere. Define the lower, right and left Dini derivatives of $V$ at $y>0$ by $D_{+} V(y)=\liminf _{\epsilon \rightarrow 0} \frac{V(y+\epsilon)-V(y)}{\epsilon}$ and $D_{-} V(y)=\liminf _{\epsilon \rightarrow 0} \frac{V(y)-V(y-\epsilon)}{\epsilon}$, and the upper, right and left Dini derivatives of V by $D^{+} V(y)=\lim _{\sup } \frac{V_{\epsilon}(y+\epsilon)-V(y)}{\epsilon}$ and $D^{-} V(y)=\limsup _{\epsilon \rightarrow 0} \frac{V(y)-V(y-\epsilon)}{\epsilon}$. Note that the the Dini derivatives can assume the values $-\infty$ or $+\infty$. To simplify notation, let $(a, x)$ be optimal from $y$. Define $W(x)=V(f(x))$. The Chain Rule for Dini Derivatives (e.g., Rockafellar and Wets (2004, Theorem 10.6) implies that the right Dini derivatives satisfy $D_{+} W(x) \geq$ $D_{+} V(f(x)) \cdot f_{x}(x)$ and $D^{+} W(x) \leq D^{+} V(f(x)) \cdot f_{x}(x)$. Similarly, the left Dini derivatives satisfy, $D_{-} W(x) \geq D_{-} V(f(x)) \cdot f_{x}(x)$ and $D^{-} W(x) \leq D^{-} V(f(x)) \cdot f_{x}(x)$. This leads to the following subsidiary Lemma.

Lemma $8.1(a) D^{+} V(y) \leq C_{y}(a, y)+\min \left[C_{a}(a, y), D_{x}(x)+\delta D^{+} V(f(x)) \cdot f_{x}(x)\right]$. (b) If $x>0$, then $C_{a}(y-x, y) \geq D_{x}(x)+\delta D_{-} V(f(x)) \cdot f_{x}(x)$ and $D_{-} V(y) \geq$ $C_{y}(a, y)+D_{x}(x)+\delta D_{-} V(f(x)) \cdot f_{x}(x)$. (c) If $a>0$, then $D_{x}(x)+\delta D^{+} V(f(x))$. $f_{x}(x) \geq C_{a}(a, y)$ and $D_{-} V(y) \geq C_{a}(a, y)+C_{y}(a, y)$.

The proof of Proposition 2.3 now follows directly from Lemma 8.1. A complete proof is provided in the working paper version (Olson and Roy 2006).

Proof of Proposition 3.1 (a) Let $\left\{x_{t}, y_{t}, a_{t}\right\}_{t=0}^{\infty}$ be optimal from $y_{0}=y$. Without loss of generality let $n=0$. Suppose $a_{t}=0$ and $x_{t}=y_{t}=f^{t}(y)>0$ for all $t$. Then the first part of Lemma 8.1(b) implies $C_{a}(0, y) \geq D_{x}(y)+\delta D_{-} V(f(y)) f_{x}(y)$. Iterating forward using $D_{-} V\left(f\left(y_{t-1}\right)\right) \geq C_{y}\left(0, y_{t}\right)+D_{x}\left(y_{t}\right)+\delta D_{-} V\left(f\left(y_{t}\right)\right) \cdot f_{x}\left(y_{t}\right)$ (see Lemma $8.1(\mathrm{~b}))$, and the fact that $C_{y}(0, y)=0$ for all $y$, it follows that $C_{a}(0, y) \geq$ $\sum_{i=0}^{\infty} \delta^{i}\left[D_{x}\left(f^{i}(y)\right) f_{x}^{i}(y)\right]$. This contradicts the condition in part (a) of the proposition. Thus, there must exist some $t$ for which $a_{t}>0$. (b) Let $\left\{x_{t}, y_{t}, a_{t}\right\}_{t=0}^{\infty}$ be optimal from $y_{0}=y$. By assumption, the system is eventually controlled from $y$, so $a_{t}>0$ for some $t$. Suppose that $a_{k}=0$ and $x_{k}=y_{k}=f^{k}(y)>0$ for $k=0,1, \ldots, t-1$, 
and $a_{t}>0$. Then, using Lemma 8.1(b) as in the proof of part (a) above, it follows that $C_{a}(0, y) \geq \sum_{k=0}^{t-1} \delta^{k} D_{x}\left(f^{k}(y)\right) f_{x}^{k}(y)+\delta^{t} D_{-} V\left(f^{t}(y)\right) f_{x}^{t}(y)$. Since $y_{t}=f^{t}(y)$ and $a_{t}>0$, Lemma 8.1(c) implies $D_{-} V\left(f^{t}(y)\right) \geq C_{a}\left(a_{t}, f^{t}(y)\right)+C_{y}\left(a_{t}, f^{t}(y)\right)$. Putting these together yields $C_{a}(0, y) \geq \sum_{k=0}^{t-1} \delta^{k} D_{x}\left(f^{k}(y)\right) f_{x}^{k}(y)+\delta^{t} \inf _{a}\left[C_{a}\left(a, f^{t}(y)\right)+\right.$ $\left.C_{y}\left(a, f^{t}(y)\right)\right] f_{x}^{t}(y)$. This contradicts the condition in part (b). (c) Since the condition in part (c) holds for all $y, C_{a}(0, f(y))<D_{x}(f(y))+\delta\left[\inf _{a}\left\{C_{a}\left(a, f^{2}(y)\right)+\right.\right.$ $\left.\left.C_{y}\left(a, f^{2}(y)\right)\right\}\right] f_{x}(f(y))$. Substituting this in the previous inequality yields $C_{a}(0, y)<$ $D_{x}(y)+\delta\left[D_{x}(f(y))+\delta\left[\inf _{a}\left\{C_{a}\left(a, f^{2}(y)\right)+C_{y}\left(a, f^{2}(y)\right)\right\}\right] f_{x}(f(y))\right] f_{x}(y)$. Iterating forward and repeating a similar substitution (note $C_{y}$ is continuous and hence bounded on $\Omega$ ) yields $C_{a}(0, y)<\sum_{t=0}^{\infty} \delta^{t}\left[D_{x}\left(f^{t}(y)\right) f_{x}^{t}(y)\right]$ which is the condition in part (a). Hence, the system is eventually controlled from $y$. Let $T$ be the first $t$ such that $a_{t}>0$ and suppose that $T>0$. Then $a_{t}=0$ and $x_{t}=y_{t}=$ $f^{t}(y)$ for $t=0, \ldots, T-1$, while $0<a_{T} \leq y_{T}=f^{T}(y)$. Lemma 8.1(b) implies $C_{a}\left(0, f^{T-1}(y)\right) \geq D_{x}\left(f^{T-1}(y)\right)+\delta D_{-} V\left(f^{T}(y)\right) \cdot f_{x}\left(f^{T-1}(y)\right)$ while Lemma 8.1(c) implies $D_{-} V\left(f^{T}(y)\right) \geq C_{a}\left(a_{T}, f^{T}(y)\right)+C_{y}\left(a_{T}, f^{T}(y)\right)$. Combining these two inequalities yields $C_{a}\left(0, f^{T-1}(y)\right) \geq D_{x}\left(f^{T-1}(y)\right)+\delta\left[C_{a}\left(a_{T}, f^{T}(y)\right)+\right.$ $\left.C_{y}\left(a_{T}, f^{T}(y)\right)\right] \cdot f_{x}\left(f^{T-1}(y)\right)$. This contradicts the condition in the proposition, so it must be that $T=0$ and $A(y)>0$ for all $y$.

Proof of Proposition 4.1 Suppose there exists $\widehat{x} \in X(y)$ such that $\widehat{x}<y$ and let $\widetilde{a} \in A(f(\widehat{x}))$. The condition in the proposition implies $0>-C_{a}(0, y)+D_{x}(y)+$ $\delta \sup _{0 \leq x<y, 0 \leq a \leq f(x)}\left[\left\{C_{a}(a, f(x))+C_{y}(a, f(x))\right\} f_{x}(x)\right] \geq-C_{a}(y-\widehat{x}, y)+D_{x}(\widehat{x})+$ $\delta\left[\left\{C_{a}(\widetilde{a}, f(\widehat{x}))+C_{y}(\widetilde{a}, f(\widehat{x}))\right\} f_{x}(\widehat{x})\right]$ where the last inequality is due to assumption B4. Since $\widehat{x}<y$, Proposition 2.3(a) implies $-C_{a}(y-\widehat{x}, y)+D_{x}(\widehat{x})+\delta\left[\left\{C_{a}(\widetilde{a}, f(\widehat{x}))+\right.\right.$ $\left.\left.C_{y}(\widetilde{a}, f(\widehat{x}))\right\} f_{x}(\widehat{x})\right] \geq 0$, a contradiction.

Proof of Proposition 4.2 For $x \in[0, y]$, define the function $H(x)=-C_{a}(0, x)+$ $D_{x}(x)+\delta\left[C_{a}(f(x), f(x))+C_{y}(f(x), f(x))\right] f_{x}(x)$. Suppose $\widehat{x} \in X(y)$ and $\widehat{x}<y$. Let $\widetilde{a} \in A(f(\widehat{x}))$. Proposition 2.3(a) and $C_{a a}+C_{a y} \geq 0$ imply $0 \leq-C_{a}(y-$ $\widehat{x}, y)+D_{x}(\widehat{x})+\delta\left[C_{a}(\widetilde{a}, f(\widehat{x}))+C_{y}(\widetilde{a}, f(\widehat{x}))\right] f_{x}(\widehat{x}) \leq-C_{a}(0, \widehat{x})+D_{x}(\widehat{x})+$ $\delta\left[C_{a}(f(\widehat{x}), f(\widehat{x}))+C_{y}(f(\widehat{x}), f(\widehat{x}))\right] f_{x}(\widehat{x})=H(\widehat{x})$. The condition in Proposition 4.2 implies $H(0)<0$. By the continuity of $H$ it follows that $H(x)<0$ for all $x$ sufficiently close to 0 . Since $\widehat{x}<y$ it must also be true that $H(\widehat{x})<0$ if $y$ is sufficiently close to zero. Thus, for all $y$ sufficiently close to zero, we obtain a contradiction.

Proof of Proposition 5.1 Suppose not. Then there exists an optimal program $\left(y_{t}, x_{t}\right.$, $\left.a_{t}\right)_{0}^{\infty}$, where $y_{1}=f\left(x_{0}\right) \geq y=y_{0}$, i.e., $x_{0} \geq f^{-1}(y)$. Since, $y_{1} \in[y, f(y)]$ and the invasion is controlled on $[y, f(y)]$, it follows that $x_{1}<y_{1}$. Therefore, using Proposition 2.3, we have $C_{a}\left(y-x_{0}, y\right) \geq D_{x}\left(x_{0}\right)+\delta\left[C_{a}\left(f\left(x_{0}\right)-x_{1}, f\left(x_{0}\right)+\right.\right.$ $\left.C_{y}\left(f\left(x_{0}\right)-x_{1}, f\left(x_{0}\right)\right)\right] f_{x}\left(x_{0}\right)$, which violates the inequality in the statement of the Proposition.

Proof of Proposition 5.2 (a) The proof follows directly from Proposition 5.1.(b) Consider $y_{0} \in(0, \zeta]$ and the optimal path $\left\{x_{t}, y_{t}\right\}$ generated by the maximal selection from $X(y)$ (as $C_{a a}(a, y)+C_{a y}(a, y) \geq 0$, this policy function is non-decreasing in $y$ ). It is sufficient to show that $y_{1}<y_{0}$ for any such $y_{0} \in(0, \zeta]$. Suppose not. 
Then, $y_{1}=f\left(x_{0}\right) \geq y_{0}$. This, in turn, implies that $x_{1} \geq x_{0}$. Since, $y_{1} \in\left[y_{0}, f\left(y_{0}\right)\right] \subset$ $(0, f(\zeta))$ where the invasion is controlled, it follows that $x_{1}<y_{1}=f\left(x_{0}\right)$. Therefore, using Proposition 2.3, we have $C_{a}\left(y_{0}-x_{0}, y_{0}\right) \geq D_{x}\left(x_{0}\right)+\delta\left[C_{a}\left(f\left(x_{0}\right)-x_{1}, f\left(x_{0}\right)\right)+\right.$ $\left.C_{y}\left(f\left(x_{0}\right)-x_{1}, f\left(x_{0}\right)\right)\right] f_{x}\left(x_{0}\right)$ and using $C_{a a}(a, y)+C_{a y}(a, y) \geq 0$ and $f\left(x_{0}\right) \geq y_{0}$ we have $C_{a}\left(f\left(x_{0}\right)-x_{0}, f\left(x_{0}\right)\right) \geq D_{x}\left(x_{0}\right)+\delta\left[C_{a}\left(f\left(x_{0}\right)-x_{1}, f\left(x_{0}\right)\right)+C_{y}\left(f\left(x_{0}\right)-\right.\right.$ $\left.\left.x_{1}, f\left(x_{0}\right)\right)\right] f_{x}\left(x_{0}\right)$, which violates the inequality in the antecedent of the proposition. (c) Consider the set of optimal paths generated by the maximal selection from $X(y)$. It is sufficient to show that complete eradication occurs on every path generated by this selection. From Lemma 2.2, we know that every optimal path generated by this selection is weakly monotone and hence convergent (they are all bounded). Suppose to the contrary that there is an optimal path generated by the maximal selection from $\mathrm{X}(\mathrm{y})$ which is bounded away from zero. Then, it must converge to a strictly positive optimal steady state $y^{*}=f\left(x^{*}\right)$, where $x^{*}$ and $y^{*}$ lie in $[0, K]$. If $x^{*} \in(0, K)$ then Proposition 2.3(c) implies $C_{a}\left(f\left(x^{*}\right)-x^{*}, f\left(x^{*}\right)\right)=D_{x}\left(x^{*}\right)+\delta\left[C_{a}\left(f\left(x^{*}\right)-\right.\right.$ $\left.\left.x^{*}, f\left(x^{*}\right)\right)+C_{y}\left(f\left(x^{*}\right)-x^{*}, f\left(x^{*}\right)\right)\right] f_{x}\left(x^{*}\right)$ which contradicts the inequality in the proposition. Also, $y^{*}=K=f(K)$ is not an optimal steady state as the inequality in the proposition implies $\left.\left.C_{a}(0, K)\right)<D_{x}(K)+\delta\left[C_{a}(0, K)\right)+C_{y}(0, K)\right] f_{x}(K)=$ $\left.D_{x}(K)+\delta C_{a}(0, K)\right) f_{x}(K)$ which implies $\left.C_{a}(0, K)\right)<D_{x}(K) /\left(1-\delta f_{x}(K)\right)$. The latter can be used to show that a program where the control is infinitesimal but greater than zero in period 0 and equal to zero every period thereafter dominates a program where $a_{t}=0$ in every period. Hence, $K$ is not an optimal steady state. Thus, it must be the case that every optimal path converges to 0 .

Proof of Proposition 5.3 Suppose not. Then there exists an optimal program $\left(y_{t}, x_{t}\right.$, $\left.a_{t}\right)_{0}^{\infty}, y_{0}=y$, where $x_{0}>0$ and $y_{1}=f\left(x_{0}\right)>0$. Since the invasion is controlled from every $y_{0} \in(0, f(y))$, it follows from Proposition 2.3 that $C_{a}\left(y-x_{0}, y\right) \geq D_{x}\left(x_{0}\right)+$ $\delta\left[C_{a}\left(f\left(x_{0}\right)-x_{1}, f\left(x_{0}\right)\right)+C_{y}\left(f\left(x_{0}\right)-x_{1}, f\left(x_{0}\right)\right] f_{x}\left(x_{0}\right)\right.$. The convexity of $C$ in its first argument and the convexity of $D$ then imply $C_{a}(y, y) \geq D_{x}(0)+\delta\left[C_{a}\left(f\left(x_{0}\right)-\right.\right.$ $\left.\left.x_{1}, f\left(x_{0}\right)\right)+C_{y}\left(f\left(x_{0}\right)-x_{1}, f\left(x_{0}\right)\right)\right] f_{x}\left(x_{0}\right)$. This contradicts the inequality in the antecedent of the proposition.

Proof of Proposition 6.1 (a) Suppose $0 \in X(y)$. Consider the alternative of increasing the remaining invasion to $\varepsilon$ and then eradicating it in the following period. By the principle of optimality $C(y, y)+D(0)+\delta[C(0,0)+D(0)]+\delta^{2} V(0) \leq C(y-$ $\varepsilon, y)+D(\varepsilon)+\delta C(f(\varepsilon), f(\varepsilon))+D(0)+\delta^{2} V(0)$. This implies $C_{a}(y, y) \leq D_{x}(0)+$ $\delta\left[C_{a}(0,0)+C_{y}(0,0)\right] f_{x}(0)=D_{x}(0)+\delta C_{a}(0,0) f_{x}(0)$, where the equality follows from $B 3$. This is a contradiction to the condition in part (a).

(b) $C_{a a}+C_{a y} \geq 0$ implies $C_{a}(0,0) \leq C_{a}(y, y)$ for all $y$. Hence the condition in Proposition 6.1(a) holds for all $y$ and $X(y)>0$ for all $y$. It now suffices to show that there exists an $\varepsilon$ sufficiently close to zero, such that $X(y)=y$ for all $y \in(0, \varepsilon)$. Let $x \in X(y)$ and suppose that $x<y$. By Proposition 2.3(a), for any $\widehat{a} \in A(f(x))$, $0 \leq-C_{a}(y-x, y)+D_{x}(x)+\delta\left[C_{a}(\widehat{a}, f(x))+C_{y}(\widehat{a}, f(x))\right] f_{x}(x) \leq-C_{a}(0, x)+$ $D_{x}(x)+\delta\left[C_{a}(f(x), f(x))+C_{y}(f(x), f(x))\right] f_{x}(x)$, using $C_{a a}+C_{a y} \geq 0$ twice. Define $H(x) \equiv-C_{a}(0, x)+D_{x}(x)+\delta\left[C_{a}(f(x), f(x))+C_{y}(f(x), f(x))\right] f_{x}(x) \geq$ 0 . The condition in part (b) implies $H(0)<0$ and by the continuity of $H$ one can 
pick an $\varepsilon$ sufficiently close to 0 such that $H(x)<0$, for all $x$ in $(0, \varepsilon)$. This yields a contradiction.

Proof of Proposition 6.2 (a) It is sufficient to show that for all $y \geq f(\widehat{x}), x \in$ $X(y)$ implies $x \geq \widehat{x}$. Suppose not. Then, there exists $y_{0} \geq f(\widehat{x})$ and an optimal path $\left\{a_{t}, x_{t}, y_{t}\right\}$ such that $x_{0}<\widehat{x}$. As $\widehat{x} \leq f(\widehat{x}) \leq y_{0}$, it must be that $x_{0}<y_{0}$. Using Proposition 2.3, we have $C_{a}\left(y_{0}-x_{0}, y_{0}\right) \leq D_{x}\left(x_{0}\right)+\delta\left[C_{a}\left(a_{1}, f\left(x_{0}\right)\right)+\right.$ $\left.C_{y}\left(a_{1}, f\left(x_{0}\right)\right)\right] f_{x}\left(x_{0}\right)$. Then $x_{0}<\widehat{x}$ implies $C_{a}\left(y_{0}-\widehat{x}, y_{0}\right) \leq C_{a}\left(y_{0}-x_{0}, y_{0}\right) \leq$ $D_{x}\left(x_{0}\right)+\delta\left[C_{a}\left(a_{1}, f\left(x_{0}\right)\right)+C_{y}\left(a_{1}, f\left(x_{0}\right)\right)\right] f_{x}\left(x_{0}\right) \leq D_{x}(\widehat{x})+\delta\left[C_{a}\left(a_{1}, f\left(x_{0}\right)\right)+\right.$ $\left.C_{y}\left(a_{1}, f\left(x_{0}\right)\right)\right] f_{x}\left(x_{0}\right)$ which violates the condition in part (a).

(b) Since the minimal selection from $X(y)$ is non-decreasing under $C_{a a}+C_{a y} \geq 0$, it is sufficient to show that every optimal path from $y_{0}=f(\widehat{x})$ generated by the minimal selection from $X(y)$ is non-decreasing. Suppose not. Then, the optimal path $\left\{x_{t}, y_{t}\right\}$ from $y_{0}=f(\widehat{x})$ generated by the minimal selection from $X(y)$ must satisfy $x_{0}<\widehat{x}$. Proposition 2.3 implies $C_{a}\left(y_{0}-x_{0}, y_{0}\right)=C_{a}\left(f(\widehat{x})-x_{0}, f(\widehat{x})\right) \leq$ $D_{x}\left(x_{0}\right)+\delta\left[C_{a}\left(a_{1}, f\left(x_{0}\right)\right)+C_{y}\left(a_{1}, f\left(x_{0}\right)\right)\right] f_{x}\left(x_{0}\right)$. As the minimal selection from $X(y)$ is non-decreasing, $x_{0}<\widehat{x}$ implies $x_{1} \leq x_{0}$. Using $C_{a a}+C_{a y} \geq 0$ twice, this implies $C_{a}\left(f\left(x_{0}\right)-x_{0}, f\left(x_{0}\right)\right) \leq C_{a}\left(f(\widehat{x})-x_{0}, f(\widehat{x})\right) \leq D_{x}\left(x_{0}\right)+\delta\left[C_{a}\left(f\left(x_{0}\right)-\right.\right.$ $\left.\left.x_{1}, f\left(x_{0}\right)\right)+C_{y}\left(f\left(x_{0}\right)-x_{1}, f\left(x_{0}\right)\right)\right] f_{x}\left(x_{0}\right) \leq D_{x}\left(x_{0}\right)+\delta\left[C_{a}\left(f\left(x_{0}\right), f\left(x_{0}\right)\right)+\right.$ $\left.C_{y}\left(f\left(x_{0}\right), f\left(x_{0}\right)\right)\right] f_{x}\left(x_{0}\right)$. This violates the condition in part (b).

\section{References}

Benveniste, L.M., Scheinkman, J.A.: On the differentiability of the value function in dynamic models of economics. Econometrica 47(3), 727-732 (1979)

Clark, C.W.: Mathematical Bioeconomics: The Optimal Management of Renewable Resources, 2nd edn. Wiley, New York (1990)

Dawid, H., Kopel, M.: On optimal cycles in dynamic programming models with convex return function. Econ. Theory 13(2), 309-327 (1999)

Dechert, W.D., Nishimura, K.: A complete characterization of optimal growth paths in an aggregated model with non-concave production function. J. Econ. Theory 31, 332-354 (1983)

Inada, K.-I.: On a two-sector model of economic growth: comments and a generalization. Rev. Econ. Stud. 30(2), 119-127 (1963)

Jaquette, D.L.: A discrete time population control model. Math. Biosciences 15, 231-252 (1972)

Majumdar, M., Mitra, T.: Intertemporal allocation with a non-convex technology. J. Econ. Theory 27, 101136 (1982)

Majumdar, M., Mitra, T.: Periodic and chaotic programs of optimal intertemporal allocation in an aggregative model with wealth effects. Econ. Theory 4(5), 649-676 (1994)

Majumdar, M., Mitra, T., Nishimura, K. (eds.): Optimization and Chaos. Springer-Verlag, Berlin (2000)

Mirman, L.J., Zilcha, I.: On optimal growth under uncertainty. J. Econ. Theory 11(3), 329-339 (1975)

Mitra, T., Roy, S.: Optimal exploitation of renewable resources under uncertainty and the extinction of species. Econ. Theory 28(1), 1-23 (2006)

Nyarko, Y., Olson, L.J.: Stochastic growth when utility depends on both consumption and the stock level. Econ. Theory 4(5), 791-797 (1994)

Olson, L.J., Roy, S.: On conservation of renewable resources with stock-dependent return and non-concave production. J. Econ. Theory 70(1), 133-157 (1996)

Olson, L.J., Roy, S.: The economics of controlling a stochastic biological invasion. AJAE 84(5), 1311$1316(2002)$

Olson, L.J., Roy, S.: The economics of controlling a biological invasion, WP03-06, Dept. of Agricultural and Resource Economics. University of Maryland, College Park (2006)

Pimentel, D., Lach, L., Zuniga, R., Morrison, D.: Environmental and economic costs of nonindigenous species in the United States. BioScience 50(1), 53-65 (2000) 
Rockafellar, R.T., Wets, R.J.-B.: Variational Analysis. Springer, Berlin (2004)

Simberloff, D.: Impacts of introduced species in the United States. Consequences 2(2), 13-22 (1996)

Strauch, R.E.: Negative dynamic programming. Ann. Math. Stat. 37(4), 871-890 (1966)

Topkis, D.M.: Minimizing a submodular function on a lattice. Oper. Res. 26, 305-321 (1978)

Vitousek, P.M., D’ Antonio, C.M., Loope, L.L., Westbrooks, R.: Biological invasions as global environmental change. Am. Scientist 84, 468-478 (1996) 